enangebot beteiligten Lehrstühle die Berufsqualifikation der Absolventen durch weitere Maßnahmen unterstiitzen. Hierzu gehören

- innovative Lehrstuhlumfelder, in denen die Unterstiitzung bei studentischer Existenzgründung, die Nutzung von neuen Medien, die Arbeit mit modernen Didaktik- und Moderationskonzepten zur Selbstverständlichkeit gehört und die den Studierenden so immer wieder Anregungen zum Nachahmen und zur Weiterentwicklung geben,

- aktive Kontaktaufnahme der Lehrstühle mit möglichen Arbeitgebern und Arbeitsfeldern der späteren Absolventen, um einerseits für die spezifischen durch den Studiengang geworbenen Qua ; lifikationen zu werben und andererseits das Ausbildungsprofil vor dem Hintergrund aktueller Ausbildungserfordernisse weiterzuentwickeln.

- gezielte Werbung und Auswahl von Studierenden für den Studiengang, um einen möglichst hohen Anteil an qualifizierten und motivierten Studierenden für den Studienschwerpunkt zu gewinnen.

- Kooperationen mit anderen Fachbereichen und Initiativen auf Universitätsebene, um das breite und qualifizierte Potential, das eine Universität im ökologischen Bereich hat, den Studierenden leichter zugänglich zu machen.

Eine transdisziplinäre ökologische Wirtschaftslehre ist keine Karrieresackgasse, wenn sie strategisch geeignet umgesetzt wird.

\section{Anmerkungen}

(1) Als prominentestes Beispiel kann hier der Studiengang Umweltnaturwissenschaften der ETH Zürich genannt werden. (2) Vgl. hierzu auch die Vorstellung des Diplomstudienganges Ökonomie mit ökologischen Schwerpunkt an der Universitöt Oldenburg in der Ausgabe Nr. 5/1997 von Ökologisches Wirtschaften sowie den Beitrag von Wenke in diesem Heft.

(3) So erst kürzlich wieder in einem Beitrag auf der Hochschulseite der Frankfurter Allgemeinen Zeitung vom 2.1.1998.

\section{Die Autoren}

Dipl.-Oec. Christoph Schwarzer ist einer der beiden Koordinatoren für den Diplomstudiengang Ökonomie mil ökologischen Schwerpunkt an der Carl-vonOssiełzky-Universitäi Oldenburg

Dr. Uwe Schneidewind vertritt die Professur für "Produktion und Umwelt" an der Universitäi Oldenburg und war zuvor an der Universiö̈t St. Gallen tätig. Kontakt: Christoph Schwarzer, Universitär Oldenburg, Fachbereich 4, Birkenweg 5, D-26111 Oldenburg, Tel:.0441/798-8265, e-mail: schwarz@unioldenburg.de. Dort ist auch eine Broschüre zum Diplomstudiengang „Ökonomie mit ökologischen Schwerpunkt" erhällich.

Transdisziplinarität in der Unternehmenspraxis

\title{
Immer bedeutenderer Schlüsselfaktor
}

\section{Transdisziplinäre Wirtschaftsforschung ist nicht nur eine wissenschaftliche \\ Trockenübung. Sie gewinnt zunehmend auch in der Unternehmensentwicklungs= und -beratungspraxis an Bedeutung. Der vorliegende Beitrag illustriert dies an zwei Beispielen aus der Marktentwicklung im Bereich Öko-Textilien sowie der Einführung von Integrierten Managementsystemen bei der Hoechst AG.}

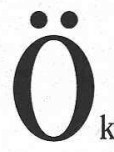

ulberwiegent kologische Produkte (1) sind derzeit Durchdringung von "Massenmärkten" gelingt nur in Ausnahmefällen. Diese Aussage ist zunächst banal, spiegelt aber die Entfernung der Wirtschaft von ökologischen Strukturen realistisch wider. Für die Initiierung ökologischer Wandlungsprozesse auf Märkten sind viele Faktoren relevant. Ein wesentliches Kriterium scheint der Versuch einer Beeinflussung von Konsumenten zu sein, der in der Regel vom unternehmerischen (Öko-)Marketing vorgenommen wird. Ökologisches Marketing, vor allem die Kommunikation mit den Konsumenten, wird häufig mit informations- ökonomischen Erkenntnissen begründet (2). Demnach liegen die Gründe für eine geringe Marktdurchdringung mit ökologischen Produkten u.a. in Informationsdefiziten. Es wird angenommen, Konsumenten seien ungenügend iiber die ökologischen Eigenschaften von Produkten informiert und gelangten aus diesem Grund nicht zu ökologisch sinnvollen Entscheidungen. Anbieter ökologischer Erzeugnisse sind da- durch gezwungen, ihre Produktmerkmale verstärkt zu kommunizieren (Signalling). Daß diese Erkenntnisse die Kommunikationspraxis ökologisch bewußter Unternehmen prägen, zeigt die zunehmend zu beobachtende Volldeklaration ökologischer Erzeugnisse. Auch hinsichtlich der Werbe-Darstellung werden ökologisch glaubwürdige Formen (Verzicht auf Hochglanzpapier, keine schrillen Werbeclips, etc.) sowie sachliche und informative Werbebotschaften empfohlen (3). Eine nähere Betrachtung zeigt jedoch, daß diese enge ökonomische Sichtweise für die Initiierung ökologischer Wandlungsprozesse ungeeignet ist.
Zum einen liegt einer solchen Handlungsempfehlung ein verkürztes, mechanistisches Kommunikationsverständnis zu Grunde. Kommunikative Prozesse werden auf ein Sender-Empfänger-Modell verkürzt, bei dem der Empfänger von Informationen (hier der Konsument) die ausgesendeten Signale einszu-eins übernimmt und folglich im Sinne des Senders handelt (4). Zudem werden Märkte zu einseitig betrachtet, da davon ausgegangen wird, daß die Konsumenten in mehr oder weniger gleichem Umfang für ökologische Belange sensibilisierbar sind. Am Institut für Wirtschaft und Ökologie der Universität St. Gallen (IWÖHSG) wird deshalb in Kooperation mit Unternehmen der deutschen und schweizerischen Bekleidungsbranche ein anderer, den interdisziplinären Notwendigkeiten einer ökologischen Marktentwicklung angemessener Weg verfolgt.

\section{Erweitertes \\ Kommunikationsverständnis}

Den Ausgangspunkt dieser Herangehensweise bildet eine soziologische Betrachtung der Rahmenbedingungen von Märkten. Die (industrialisierten) Gesellschaften tendieren - vereinfacht gesagt - aufgrund von Aspekten wie Enttraditionalisierung (5), Multioptionalisierung (6) oder Erlebnisorientierung (7) in Richtung einer starken gruppen- und individualspezifischen Fragmentierung. Daraus folgt letztlich, daß es nicht den Massenmarkt für Produkte gibt, sondern nur stark fragmentierte Teilmärkte.

Auch kommunikationstheoretisch wird mit einem anderen Ansatz gearbeitet. Kommunikation ist ein sozialer Prozeß, in dem die beteiligten Akteure mittels interpretierbarer Worte und Taten Informationen mit einer bestimmten Intention zu transportieren versuchen. Dieser 
Interpretationsspielraum läßt Kommunikation weniger mechanistisch werden, sondern stellt sie auf lerntheoretische, dialogische und sprachkulturelle Fundamente (8).

Aus diesen beiden Ansätzen resultiert, daß Marktkommunikation (9) als Zusammenspiel soziologischer, ökonomischer, kultureller, psychologischer und erkenntnistheoretischer As-pekte begriffen werden muß (10) (vgl. Abbildung). Sie kann nur dann annähernd er-folgreich sein, wenn die ,kommunizierende Unternehmung“ die Wahrnehmungsmuster ihrer Adressaten erkennt, versteht und berücksichtigt.

Marktkommunikation beinhaltet folglich nicht nur Werbemaßnahmen im engen Sinne, sondern auch die Gestaltung von Produkten und Preisen oder die Berücksichtigung produktkultureller (11) Faktoren. Damit wird über den im Marketing verwendeten Begriff hinausgegangen. Kommunikationsaktivitäten in fragmentierten Märkten müssen deshalb zielgruppenadäquat sein, sofern eine stärkere Durchdringung mit ökologischen Erzeugnissen gelingen soll. In dem am IWÖ-HSG untersuchten Bekleidungsbereich lassen sich diese Erfordernisse besonders deutlich darstellen, da er sich durch verschiedene subkulturelle Strömungen auszeichnet, unter denen die „ökologische" Richtung nur nebensächlich ist. Dies zeigt sich zum einen anhand des Umsatzanteils ökologisch hergestellter Textilien am Gesamtbranchenumsatz, der bei weit unter einem Prozent liegt. Zum anderen stellen dies auch empirische Studien zum Konsumverhalten heraus, die belegen, daß ,Ökologie“ und „Gesundheit" im textilbezogenen Entscheidungsprozeß der Konsumenten nur geringe Bedeutung haben und zum Teil gar Vorurteile bewirken (12). Der am IWÖ-HSG entwickelte Ansatz ist deutlich weniger "fundamentalistisch" als herkömmliche Öko-Marketing- Empfehlungen, weil er nicht versucht, Produkte für beliebige Zielgruppen entsprechend des ,ökologischen Optimums" herzustellen und zu kommunizieren. Statt dessen lehnt er sich an das unter Berücksichtigung der Wahrnehmungsprioritäten des jeweiligen Konsumentensegments „Mögliche“ an. Dies kann dann, um auf die informationsökonomischen Empfehlungen zu Beginn des Beitrags zurückzukommen, beispielsweise im Segment (Öko-)Kleidung für jugendliche Subkulturen in einer höchstens dezenten Information über ökologische Produktvorzüge bestehen.

Abb.: Markłkommunikation als fransdisziplinäres Erfordernis

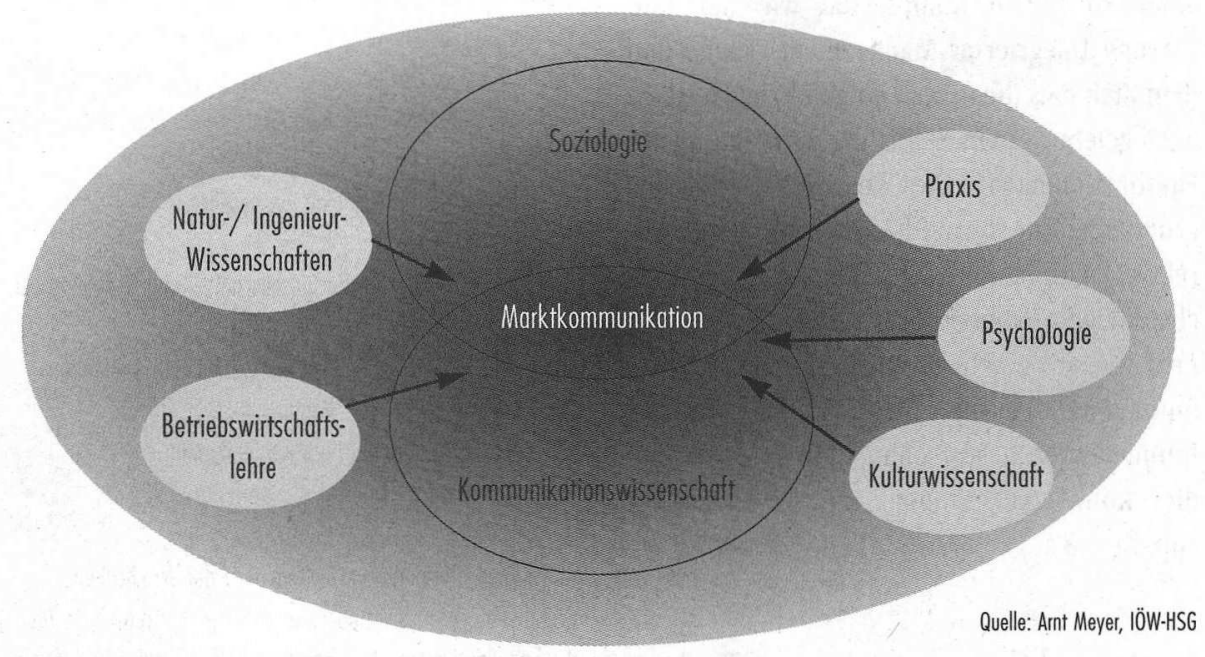

\section{Transdisziplinäre Organisationsentwicklung}

Transdisziplinäre ökologische Beratungsansätze spielen dabei nicht nur in der UnternehmenMarkt-Beziehung eine wichtige Rolle. Auch in der Organisationsentwicklung von Unternehmen ist insbesondere der Rückgriff auf psychologische und (industrie)soziologische Erkenntnisse bedeutsam (13).

Bei den Unternehmen der Hoechst AG am Standort Frankfurt-Höchst wurden in den letzten zwei Jahren Integrierte Managementsysteme unter bewußter Berücksichtigung solcher interdisziplinären Bezüge eingeführt. Hierbei werden die Managementsysteme für Umwelt, Qualität, Sicherheit und Gesundheitsschutz zusammengeführt. Gründe für die Integration dieser Systeme sind das Erreichen von Effizienz-, Sicherungsund Innovationszielen (14), die Integration leistet zudem einen wichtigen Beitrag zur Organisationsentwicklung in Richtung einer umwelt-, qualitäts- und sicherheitsorientierten Unternehmensorganisation. Dabei sollen Integrierte Managementsysteme Integrationskraft besitzen, einfach, verständlich und akzeptierbar sein. Um diese Anforderungen in der Praxis zu verwirklichen und ein gelebtes System zu implementieren, ist neben den Struktur- und Systemfragen bei der Umsetzung auch die Verhaltensebene zu berücksichtigen.

Hierzu wurden u.a. Instrumente des Management of Change (das Vier-Ebenen-Modell, Umgang mit Widerständen,...), die in Projekten der Unternehmensentwicklung erforscht wur- den, eingesetzt. Die Entwicklung und Anpassung dieser Instrumente auf die Hoechst-Situation und das einzusetzende Projekt erfolgte in einem interdisziplinären Team (Naturwissenschaftler, Ingenieure, Industriesoziologen, Wirtschaftswissenschaftler, Pädagogen und Psychologen).

So wurden spezielle Schulungen für die Projektleiter entwickelt, welche sowohl die fachlichen Inhalte Integrierter Managementsysteme beinhalteten, als auch die verhaltensbedingten Aspekte der Implementierung. Hierzu ist z.B. das Vier-Ebenen-Modell vorgestellt und eingesetzt worden, mit welchem sowohl die Sachebene mit den Meilensteinen, als auch die psychosoziale Ebene geplant und somit mögliche Problemfelder und Widerstände vom Projektteam erkannt und proaktiv angegangen werden können.

Bei der Umsetzung hat sich gezeigt, daß viele Probleme beim Aufbau und der Umsetzung der Integrierten Managmentsysteme in Unternehmen mit dem Einsatz dieser Instrumente im Vorfeld erkannt werden konnten. Die verstärkte Betrachtung des Faktors Mensch bei der Umsetzung hat zur Einbindung aller Mitarbeiterschichten, zielgruppenspezifischeren Weiterbildungs-, Schulungs- und Motivationsmaßnahmen, besserer Beachtung der Mikropolitik (Managementsupport, Machtkämpfe, Interessenskonflikte, Widerstände) sowie dem Finden von Promotoren (Fach-, Macht- und Prozeßpromotoren) geführt.

Traditionell stehen die Struktur des Systems, die fachinhaltlichen Probleme oder die technischen Fragen im Mittelpunkt der Betrachtung. 
Bei einem Implementierungsvorhaben mit einer so hohen Komplexität wie der Einführung Integrierter Managementsysteme und dem Ziel, daß die formal eingeführten Systeme auch gelebt werden, bildet die Betrachtung des Faktors Mensch eine wesentliche Erfolgsgrundlage. Um sowohl für die Lösung struktureller, fachinhaltlicher, technischer und psycho-sozialer Probleme gerüstet zu sein, ist das Arbeiten in einem interdisziplinären Team unumgänglich, das die Erkenntnisse der Implementierungsforschung immer wieder auf die konkreten Fragen im Praxiskontext anpaßt.

\section{> Perspektiven}

Die beiden vorgestellten Beispiele zeigen, daß sowohl die Erweiterung ökologischer Handlungsfreiräume von Unternehmen als auch die Umsetzung von ökologischen Belangen in der Unternehmung Wissen benötigt, das disziplinäre Grenzen sprengt. Heute sind es in der Regel erst Pionierunternehmen, die transdisziplinäre Transformationsansätze konsequent umsetzen. Für die ökologische Unternehmensberatung und -entwicklung der Zukunft wird die Mobilisierung eines problemorientierten interdisziplinären Wissens zu einem immer bedeutenderen Schlüsselfaktor werden.

\section{Anmerkungen}

(1) Unter einem ökologischen Produkt soll ein im Vergleich zu anderen Produkten mit ähnlichem oder gleichem "Nutzen" über den gesamten ökologischen Produktlebenszyklus verbessertes Erzeugnis verstanden werden.

(2) Vgl. K.P. Kaas: Informationsprobleme auf Märkten für umwelffreundliche Produkte. In: G.R. Wagner (Hrsg.): Betriebswirtschaft und Umweltschutz. Stuttgart 1993, S. 29-43 oder A. Hüser, C. Mühlerkamp: Werbung für ökologische Güter. Gestaltungsaspekte aus informationsökonomischer Sicht. In: Marketing ZFP, Heft 3, III/ 1992,

S. 149-156.

(3) Vgl. H. Meffert, M. Kirchgeorg: Marktorientiertes Umweltmanagement. Grundlagen und Fallstudien. Stuttgart 1993, S. 230.

(4) Das Marketing bezieht dieses Verständnis von Kommunikation im wesentlichen aus dem weiterentwickelten mathematischen Kommunikationsmodell von Shannon/Weaver. Vgl. C.E. Shannon, W. Weaver: The Mathematical Theory of Communication. Urbana, II. University of Illinois Press, 12. Aufl. 1971. Vgl. zur Kritik auch, D. Fischer, B. Kühling, Pfriem, R., Schwarzer, C.: Kommunikation zwischen Unternehmen und Gesellschaft. Schriftenreihe Nr. 4 des Lehrstuhls für Allgemeine Betriebswirtschaftslehre, Unter- nehmensführung und betriebliche Umweltpolitik, 2. Aufl., Oldenburg 1995, S. $39 \mathrm{ff}$.

(5) Vgl. A. Giddens: Leben in einer posttraditionalen Gesellschaft, in: U. Beck, A. Giddens, S. Lash (Hrsg.): Reflexive Modernisierung. Eine Kontroverse. Frankfurt 1996.

(6) Vgl. P. Gross: Die Multioptionsgesellschaft. Frankfurt 1994.

(7) Vgl. G. Schulze: Erlebnisgesellschaft. Kultursoziologie der Gegenwart. 6. Aufl., Frankfurt 1996.

(8) Hinter diesem Kommunikationsverständnis stehen vor allem die Theorien von P Watzlawick., J.H. Beavin, D.D. Jackson, : Menschliche Kommunikation - Formen, Störungen, Paradoxien. 9. Aufl., Bern u.a. 1996 und von

B. Vaassen: Die narrative Gestalt(ung) der Wirklichkeit. Grundlinien einer postmodern orientierten Epistemologie für die Sozialwissenschaften. Bamberg 1994 (Zugl. Dissertation Nr. 1508 an der Universität St. Gallen).

(9) Der Begriff ist von der anders gelagerten Anspruchsgruppenkommunikation zu unterscheiden, da es nicht um Umweltberichterstattung oder Dialogmanagement geht, sondern um die Kommunikation in den Absatzmarkt hinein.

(10) Vgl. A. Meyer: Unternehmerische Marktkommunikation im ökologischen Kontext. Ökologisch orientierte Bekleidung für den "Massenmarkt". IWÖ-Diskussionsbeitrag, St. Gallen (erscheint voraussichtlich Anfang 1998).

(11) Vgl. R. Eisendle, E. Miklautz: Produktkulturen. Dynamik und Bedeutungswandel des Konsums. Frankfurt 1992.

(12) Vgl. Schweizer Baumwollinstitut: Die ökologische Herausforderung. Theorie und Praxis beim Kauf von Textilien. Zürich 1992.

(13) Vgl. vor diesem Hintergrund insbesondere die Bücher von C. Burschel: Umweltschutz als sozialer Prozeß. Opladen 1996, sowie das gerade erschienene Handbuch Umweltschutz und Organisation von M. Birke, C. Burschel, M. Schwarz, München und Wien 1997.

(14) T. Dyllick: Von der Debatte EMAS vs. ISO 14001 zur Integration von Managementsystemen, in: UmweltWirtschaftsforum, 5. Jg (1997), H.1., S. 3. Vgl. weiterhin zu Zielen von Integrierten Managementsystemen: R. Felix, A. Pischon, F. Riemenschneider., H. Schwertle: Integrierte Managementsysteme, IWÖ-HSG-Diskussionsbeitrag Nr. 41, St. Gallen 1997, S. 3.

\section{Die Autoren}

Dipl.-Kfm. Arnt Meyer ist wissenschaftlicher Mitarbeiter am Institut für Wirischaft und Ökologie an der Universilät St. Gallen (IWÖ-HSG).

Dipl.-Kfm. Frank Riemenschneider unterstüızt bei der Hoechst-Tochter Infraserv die Einführung Intergrierter Managementsysteme im Hoechst-Konzern.

Kontakt: Arnt Meyer, IWÖ-HSG, Tigerbergstr. 2, CH-9000 St. Gallen, Tel: $++41 / 71 / 224-2739$, e-mail: Arnt.Meyer@iwo.unisg.ch
Literatur zum Schwerpunkf

\section{Bücher/Reader}

- Akademie der Wissenschaften zu Berlin: Einheit

der Wissenschaften: internationales Kolloquium de Akademie der Wissenschaften zu Berlin, Bonn, 25-27. Juni 1990, Berlin, New York 1991.

- Balsiger, P. W., Defila, R., Di Giulio, A. (Hrsg.):

Ökologie und Interdisziplinarität - eine

Beziehung mit Zukunft?, Bosel 1996.

- Epton, S.R., Payne, R.L., Pearson, A.W. Managing

interdisciplinary Research, Chister, New York u.o. 1983.

- Gräfrath, B., Huber, R., Uhlemann, B.: Einheit,

Interdisziplinarität, Komplementarität: Orien-

tierungsprobleme der Wissenschaft heute, Aka-

demie der Wissenschaften von Berlin, Forschungsbericht

3, Berlin und New York 1991.

- Hirsch, G., Hungerbühler, K., Koller, T.: Transdis-

ziplinäre Forschung - was ist damit

gemeint?, in ETH intern, No. 9/96 und 97,

S. 6-7, Zürich 1996/97.

- Holzhey, H. (Hrsg.), Interdisziplinär,

Basel und Stuttgart 1974.

- Kocka, J. (Hrsg.): Interdisziplinarität, Proxis - Herausforderung - Ideologie, Suhrkamp, Frankfurt am Moin 1987.

- Schwerpunktprogramm Umwelt des Schweizerischen Nationalfonds: Panorama "Transdisziplinarität", Nr.5/95, Bern 1995.

- Stiftung Mensch - Gesellschaft - Umwelt: Inferdisziplinarität, Arbeitspapier MGU Nr. 12/96, Basel 1996.

- Strehle, A.: Stufen sozialwissenschaftlicher Integration. Darstellung und Kritik bestehender und never Konzepte zur Integration der Sozialwissenschaften. Eine Vergleichsstudie, Dissertation an der Hochschule St. Gallen 1978.

\section{Aufsätze}

- Defila, R., Di Gulio, A. Interdisziplinäre Forschungsprozesse, in: Koufmann-Hayoz, R., Di Gulio, A. Hrsg. : Umweltproblem Mensch, Bern 1996.

- Hirsch, G.: Beziehungen zwischen Umweltforschung und disziplinürer Forschung, in: GAIA No. 5-6/1995, S. $302-314$.

- Mittelstrab, J.: Interdisziplinaritüt oder Transdisziplinarität?, in: Hieber, L. (Hrsg.): Utopie Wissenschaft. München 1993.

- Schurz, R.: Ist Inferdisziplinarität möglich?, in: Universitas, Stuttgart 1995, S. 1080-1089.

- Ulich, H.: Die Betriebswirtschaftslehre als anwendungsorientierte Sozialwissenschaft, in: Ulich, H.: Management. Herousgegeben von Dyllick, T., Probst, G.J.B., Bern und Stuttgart 1984, S. 168-200. 
(c) 20I0 Authors; licensee IÖW and oekom verlag. This is an article distributed under the terms of the Creative Commons Attribution Non-Commercial No Derivates License (http://creativecommons.org/licenses/by-nc-nd/3.o/), which permits unrestricted use, distribution, and reproduction in any medium, provided the original work is properly cited. 